\section{Der suchtkranke Patient in der Anästhesie Risiken erkennen und vorbeugen}

Screening, Diagnose und Behandlung Alkoholkonsumbezogener Störungen

Seite 406

Patienten mit Benzodiazepinabhängigkeit

Seite 416

Postoperative Überwachung und Behandlung
Thomas Hachenberg

Am 21. Mai 2015 wurde der Drogen- und Suchtbericht 2015 der Bundesregierung vorgelegt. Er enthält zahlreiche wichtige Informationen zu einem großen Spektrum an legalen und illegalen Substanzen, darunter Alkohol, Tabak, Cannabis, Amphetamine bis hin zu Heroin und ist abrufbar unter www.drogenbeauftragte.de. Auch aus anästhesiologischer und intensivmedizinischer Sicht sind diese aktuellen Ergebnisse von großem Interesse, da suchtkranke Patienten mit einer deutlich erhöhten Morbidität und Letalität belastet sind. Die Bedeutung der Problematik soll schlaglichtartig anhand einiger Daten aus dem Drogen- und Suchtbericht sowie verschiedener aktueller Studien aus Deutschland erläutert werden.

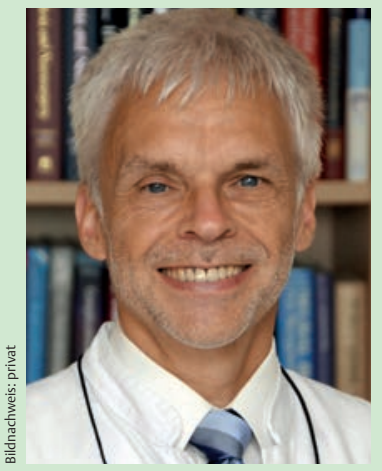

Prof. Dr. Dr. med. Thomas Hachenberg ist seit 2001 Direk tor der Klinik für Anästhesiologie und Intensivtherapie der Universität Magdeburg. Im BDA vertritt er die Universitätsanästhesisten. Prof. Hachenberg ist Landesvorsitzender der DGAI in Sachsen-Anhalt und 1. Sprecher des wissenschaftlichen Arbeitskreises „Anästhesie in der Thoraxchirurgie“. E-Mail: Thomas. Hachenberg@med.ovgu.de
Alkoholkonsum weit verbreitet Seit 1980 werden vom Institut für Therapie- und Gesundheitsforschung (IFT) München Repräsentativerhebungen zum Substanzkonsums in Deutschland in der Allgemeinbevölkerung (18-64 Jahre) durchgeführt und als Epidemiologischer Suchtsurvey (ESA) publiziert ( 0 www.esa-survey.de). Nach diesen Daten hat nur eine sehr kleine Gruppe der Erwachsenen in Deutschland (3,6\%) nie Alkohol konsumiert. Weitere 9,8\% lebten in den vergangen 12 Monaten vor der Erhebung abstinent, haben aber Erfahrungen mit Alkohol. Die große Mehrzahl (71,5\%) trank nach ESA innerhalb der letzten 30 Tage Alkohol.

Riskanter Konsum Bemerkenswert ist aber die Tatsache, dass immerhin 14,2\% der Erwachsenen zu den riskant Konsumierenden gerechnet werden müssen. Dabei ist der Anteil der Personen mit 4 oder mehr Rauschtagen (definiert als Konsum von 5 oder mehr alkoholischen Getränken pro Tag) erheblich. Nach den Studien des IFT München konsumieren in Deutschland 9,5 Mio. Menschen im Alter von 18-64 Jahren in gesundheitlich riskantem Ausmaß Alkohol, 1,77 Mio. gelten als alkoholabhängig und bei 1,6 Mio. liegt ein Alkoholmissbrauch vor. Da gerade bei Suchterkran-

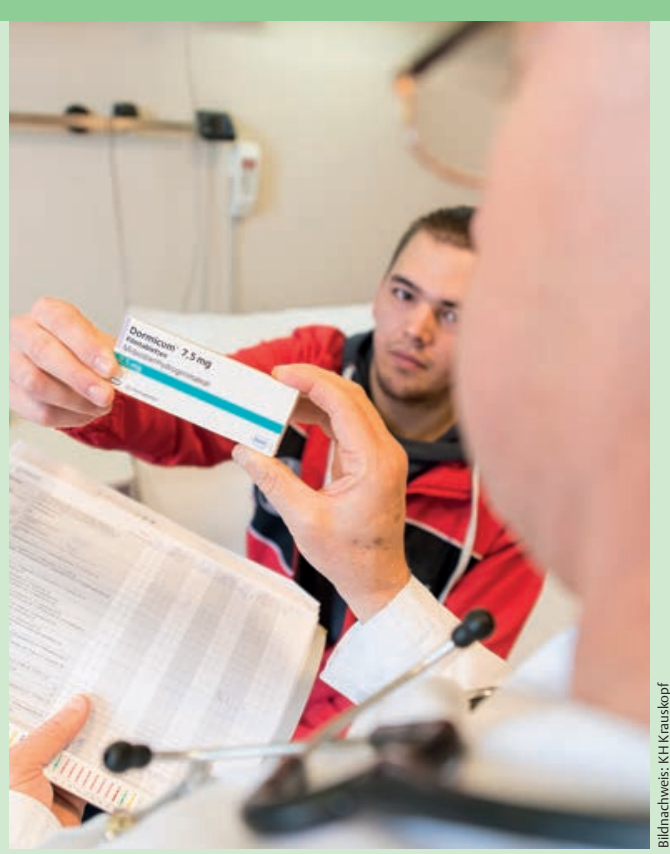

kungen eine große Dunkelziffer besteht, sind diese Daten wahrscheinlich eine konservative Annahme.

- Laut ESA wird davon ausgegangen, dass pro Jahr 74000 Menschen an den Folgen des Alkoholkonsums versterben,

davon ca. $24 \%$ durch den Alkohol allein und ca. $76 \%$ durch den kombinierten Konsum von Alkohol und Tabak. Patienten mit Polytrauma oder Tumoren des Gastrointestinaltrakts weisen in mind. 50\% einen riskanten Alkoholkonsum auf oder sind als suchtkrank zu betrachten. Während seit 1995 positive Entwicklungen bei Männern zu weniger Alkoholkonsum oder höherer Abstinenz beobachtet werden, nimmt die Prävalenz der Abhängigkeit bei jungen Frauen bis 24 Jahren zu.

Alkoholassoziierte Krankheitsfälle Das IFT führte auf der Basis einer WHO-Empfehlung eine Studie zur Krankheitslast und Sterblichkeit im Zusammenhang mit Alkoholkonsum in Deutschland durch. Demnach erhöhte sich in den Jahren 2000-2012 die Anzahl der alkoholassoziierten Krankenhausfälle bei Frauen um 38\% und bei Männern um $16 \%$. Als erfreulicher Trend war ein Rückgang der alkoholassoziierten Mortalität in allen Krankheitskategorien um 33\% zu verzeich- 
nen. Dies kann Ausdruck einer verbesserten Diagnostik und Behandlung bei Patienten mit riskantem Alkoholkonsum, mit Alkoholabhängigkeit oder-missbrauch sein.

Die aktuelle Datenlage zur Erkennung und Behandlung typischer alkoholassoziierter Komplikationen fasst Tim Neumann im ersten Topthemabeitrag zusammen. Durch den engen Bezug zur derzeit aktuellen Leitlinie „Alkoholbezogene Störungen: Screening, Diagnose und Behandlung“" werden die Empfehlungen auf eine breite Grundlage gestellt.

Medikamentenmissbrauch/-abhängigkeit In der anästhesiologischen und intensivmedizinischen Praxis spielt jedoch häufig der Konsum anderer Substanzen eine erhebliche Rolle, z.B. von Benzodiazepinen. Hierzu ist die Datenlage weniger gut. Vor allem Schlaf-, Beruhigungs - und Schmerzmittel besitzen ein häufig unterschätztes Suchtpotenzial. Diese beeinträchtigen nicht nur die kognitive Leistungsfähigkeit insbesondere bei älteren Menschen, sondern sind auch mit einer Vielzahl operativer und nicht operativer Krankheitsbildern assoziiert. Nach den Daten des ESA 2012 nehmen 4,2\% der Erwachsenen im Alter von 18-64 Jahren mind. einmal wöchentlich Antidepressiva und Analgetika, 1,2\% Beruhigungsmittel (überwiegend Benzodiazepine), $1 \%$ Neuroleptika und $0,8 \%$ Schlafmittel. Nach DSM-IV (DSM= Diagnostic and Statistical Manual of Mental Disorders) besteht eine Abhängigkeit für Schmerzmittel bei ca. $3,4 \%$, für Beruhigungsmittel bei $1,4 \%$ und für Schlafmittel für 0,8\% der Personen.

- Zusammengefasst sind etwa 2,31 Mio. Menschen in Deutschland von mind. einer der 3 Arzneimittelgruppen abhängig und weitere 4,61 Mio. weisen einen Missbrauch auf.

Relevantes klinisches Problem Auch wenn man berücksichtigt, dass z.B. das Robert KochInstitut zu etwas niedrigeren Zahlen kommt, zeigt die Datenlage eindeutig, dass Medikamentenmissbrauch und -abhängigkeit ein sehr relevantes klinisches Problem darstellen. Dementsprechend müssen sich Anästhesisten im perioperativen Bereich darauf einstellen, ein mögliches Risiko zu erkennen und eine adäquate Prophylaxe und Therapie durchzuführen. Im Beitrag von Christine Schneemilch und Michael Brinkers wird der Fokus auf Abhängigkeit von Benzodiazepinen gelegt. Dabei spielt nicht nur der Missbrauch eine Rolle, sondern auch in erheblichem Maße eine Entzugssymptomatik mit mnestischen Störungen, Desorientiertheit und Delirium tremens nach Langzeiteinnahme.

Postoperative Überwachung und Behandlung Dem Thema der postoperativen Überwachung und Behandlung des suchtkranken Patienten widmet sich die Übersichtsarbeit von Caren Tietz und Christof Strang. Hierbei rücken mehrere
Krankheitsbilder in den Mittelpunkt. Unstrittig betrifft die Problematik am häufigsten Patienten mit riskantem Alkoholkonsum, Alkoholmissbrauch oder -abhängigkeit. Auf die Besonderheiten von Benzodiazepinmissbrauch wurde bereits hingewiesen.

Illegale Drogen Zahlenmäßig anders, aber klinisch von hoher Bedeutung stellt sich der Konsum illegaler Drogen dar. Hierzu zählen v. a. Cannabis, Amphetamine, LSD, Opioide, Kokain/Crack, aber auch neue psychoaktive, meist synthetische Stoffe, die als Designerdrogen, Research Chemicals oder Legal Highs bezeichnet werden.

Der geschätzte Prozentwert für Cannabismissbrauch - die nach wie vor am häufigsten konsumierte illegale Droge - liegt bei 0,5\% der untersuchten Bevölkerung. Mit deutlichem Abstand folgen Kokain $(0,2 \%)$ und Amphetamine $(0,1 \%)$. Nach den Ergebnissen der Bundeszentrale für gesundheitliche Aufklärung weisen ca. 280000 Menschen einen Missbrauch und fast 320000 Menschen eine Abhängigkeit von mind. einer der 3 Substanzen auf. Bei Opioidsuchtkranken wird von ca. 100000-150000 Personen in Deutschland ausgegangen, davon $90 \%$ mit einer Heroinsucht. Bei etwa 78000 Personen erfolgte im Jahr 2014 eine Substitutionsbehandlung, vorwiegend mit Methadon.

Anästhesiologische Aspekte Aus anästhesiologischer Sicht sind die Ergebnisse der DRUCKStudie (Drogen und chronische Infektionskrankheiten) des Robert Koch-Instituts bedeutsam. In einem Sero- und Verhaltenssurvey vom April 2012 bis März 2015 wurden in 8 Studienstädten Informationen zu Infektionen und Verhaltensweisen von Menschen ( $n=2077$ ) erfasst, die aktuell Drogen spritzten. Der Infektionsstatus zeigt eine Prävalenz von 0-9,1\% für HIV, 42,3-75\% für HCV (abgelaufene oder aktive/chronische Infektion) und $0,3-3 \%$ HBV positiv (aktive/chronische Infektion), wobei 15,9-69,2\% HBV suszeptibel, d.h. weder geimpft noch infiziert eingeschätzt wurden.

Neben diesen klinisch-infektiologischen Problemen spielt selbstverständlich die Suchtproblematik eine große Rolle. Vor allem bei Notfalloperationen treten immer wieder opioidabhängige Patienten auf, bei denen entweder der Konsum oder eine Substitutionsbehandlung besondere Berücksichtigung erfordert.

Alle 3 Topthemabeiträge beleuchten eine aktuelle klinische Problematik, die in besonderem Maße eine sach- und fachkundige und v.a. individuell an die jeweilige Suchterkrankung angepasste Vorgehensweise erfordert.

Ihr

Thomas Hachenberg
Beitrag online zu finden unter http://dx.doi.org/ $10.1055 / \mathrm{s}-0041-102878$ 